\title{
Dangers of Adhering to an Obsolete Paradigm: Could Zika Virus Lead to a Reversal of Human Evolution?
}

\author{
Wickramasinghe $\mathrm{NC}^{1,2}$ and Steele $\mathrm{EJ}^{3^{*}}$ \\ ${ }^{1}$ Buckingham Centre for Astrobiology (BCAB), Buckingham University, UK \\ ${ }^{2}$ Institute for the Study of Panspermia and Astroeconomics, Gifu, Japan \\ ${ }^{3}$ CY O'Connor ERADE Village Foundation, PO Box 5100, Canning Vale South 6155, WA, Australia
}

*Corresponding author: Steele EJ, CY O'Connor ERADE Village Foundation, PO Box 5100, Canning Vale South 6155, WA, Australia, Tel: +61 8 9397 1556; E-mail: ejsteele@cyo.edu.au

Received date: Feb 06, 2016; Accepted date: Feb 11, 2016; Published date: Feb15, 2016

Copyright: ( 2016 Wickramasinghe NC, et al. This is an open-access article distributed under the terms of the Creative Commons Attribution License, which permits unrestricted use, distribution, and reproduction in any medium, provided the original author and source are credited.

\begin{abstract}
The recent emergence of a form of the Zika virus leading to microencephaly in new-born infants would continue to pose a major Global threat until its ultimate origins are properly understood. We argue that adhering to the obsolete paradigm of closed-box Darwinian evolution is a major obstacle to progress. If the Zika virus escalates unchecked its soma-to-germ line transfer might effectively lead to the emergence of a new human species with a diminished level of cognition.
\end{abstract}

Keywords: Zika virus; Darwinian evolution; Cosmic origins of life; Panspermia; Lamarckism; Soma-to-germline gene transfer; Ancestral haplotypes

\section{Preamble}

Darwinian Theory, based on random genetic mutations and natural selection in a closed system, continues to be invoked as the sole mechanism to explain biological evolution. Whilst ongoing evolution based only on neo-Darwinism has been amply verified at a molecular genetic level in bacterial cell cultures, for example, its extension to macro-evolution is without evidential support. This dogma has outlived its utility and now requires emphatic refutation and major revision based on the accumulated evidence from observation, analysis and experiment over the past 50 years. We fear that continued adherence to such a manifestly wrong theory places mankind at great risk, unable to either understand or rationally respond to current and future biomedical challenges.

\section{Panspermia, Evolutionary Bursts and Genome Structure}

There remain many unanswered questions pertaining to sudden phenotype changes - bursts of speciation, as evidenced in the Metazoan explosion 540 million years ago, and also periodic episodes of mass extinction [1-3]. Fred Hoyle and one of the present authors (NCW) argued from 1980 onwards that new genetic information derived from viruses plays a major role in the onward sweep of evolution. These include abrupt emergence of orders, genera and species as evidenced in the fossil record [4].

Over the past two decades there has been a growing recognition that horizontal gene transfer, via the agency of viruses, might play a key role in evolution. One of us [5] recently reviewed this data and argued that the process of horizontal gene transfer should be extended well outside our planet's biosphere. With current estimates of over 100 billion habitable planets in our galaxy alone [6] such an extension, admitting the concepts of panspermia, would seem entirely reasonable. Over the past decade there has been growing evidence (still being refuted) for microorganisms currently entering the stratosphere of the Earth $[1,2]$. Ignoring this possibility of a continuing input of microbial and viral genes to the Earth from the external universe might be fraught with imminent danger.

As discussed elsewhere [7] the 1918-1919 "Spanish Flu" Pandemic occurred before the advent of air travel and its spread had many inconsistent epidemiological features at odds with a human-to-human contagion explanation, the most striking being the simultaneous outbreaks of the disease in far distant global locations, such as Boston and Bombay, on the same day [8-11]. Such inconsistent pathogen transmission phenomena, when carefully evaluated, may apply to other historical pandemics [9]. A logical explanation of all the data invokes novel pathogenic bacteria and viruses arriving from space simultaneously in different parts of the globe thus infecting susceptible animals, insects and humans. Interpretations like this need also apply to recent historical episodes [12] despite the complications of air travel clouding the pattern of initial viral spread viz. the explosive and unexpected emergence of viral epidemics and pandemics involving HIV, Ebola, SARS and now Zika which have systematically left modern organized medicine and health authorities flat-footed.

The human genome, as well as the genomes of other life-forms, have recently been sequenced and found to contain over $8 \%$ of sequences identified to be of retroviral origin (ERVs). What this shows clearly is that humans (and other forms of life) are all survivors of a long chain of viral infections that have influenced the structure, evolution and organization of the genome $[5,13]$. Conservative recent estimates indicate a large number of non-retroviral sequences of wide-ranging viral genera have been integrated in many animal genomes (mammals, birds, insect vectors). These date at least to 30,68 and 93 million years ago, with integrations apparently assisted by the reverse transcriptases encoded in LINE retro-elements, and some viral genes are still being used (exapted) in cellular functions [14,15]. Plant genomes have not been spared such non-retroviral invasions [16]. 
In this connection the most striking feature when one analyses genome DNA sequence structure - the forest rather than the trees - is the repeat sequence background (e.g. detected using the RepeatMasker alignment tool at the UCSC Browser) against which protein-coding genes are conventionally embedded. Further, this is most dramatically observed when genomic regions are aligned against themselves using Dot Plotting methods to search for nearby duplicated segments, both tandem and inverted. Such a procedure reveals a high density (an all pervasive background throughout the genome) of short (100 nt) and long (1000 nt-10,000 nt) repetitive elements (most are assumed to be retro-elements). However with the recent discovery (above) of numerous non-retroviral genomic viral invasions it begs the question: How much more dense might this repeat background be if the alignment software of web browsers were updated to include characteristic sequence motifs of all these other viral groups. With respect to gene numbers and proportions, the human genome carries information for about 20,000-25,000 protein-coding genes or about $2 \%$ of the genome [17]. It is generally agreed that about $40 \%$ of the genome encodes for retroviruses and retro-elements when these are all added up. What then of the other $50 \%$ of the genome? One widely held (and often tacit) view is it encodes yet to be discovered RNA-based gene regulatory sequences. This may turn out to be true. Another possibility is it encodes sequences of all those other non-retro RNA viruses which have been assisted in their integration by the ubiquitous reverse transcriptases encoded by LINE elements. These viral-derived sequences are indeed likely to contribute significantly to gene regulation and expression.

One of us (EJS) recently evaluated the evidence for Panspermia independently assembled by co-author NCW and his associates, principally with Fred Hoyle, over the past 40 years [1,2]. In EJS's considered opinion the weight of evidence for the H-W Panspermia theory is on such a scale and so overwhelming in its specific details that it would easily convince a jury of objective scientists if not ordinary citizens.

We are staggered by the fact that in the 21 st Century this multifactorial and decisive evidence is not being taken seriously by main stream biomedical and scientific thinking or by the Centres for Disease Control in Atlanta and The Pirbright Institute in Woking. If the tacit stumbling block is emotional, and thus a reluctance to remove the word "Darwinian" from the evolutionary lexicon, then one alternative is to admit that Darwinian evolutionary mechanisms, involving all types of adaptation, gene transfer and selective survival has been ongoing on a Cosmic scale since time immemorial dating back 13 billion years to the earliest vestiges of the visible Universe.

\section{Soma-to-Germline Feedback}

In another related evolutionary context EJS has spent 40 years developing the concepts and assembling the evidence for Lamarckian soma-to-germline gene feed-back in both somatic cells and germ cells [18-26]. This work was recognized at the outset as clearly nonDarwinian in contradiction of the Weismann dogma (the 19th Century notion of a hypothetical barrier protecting germ cells from potentially disruptive mutated cellular genes arriving continuously from the soma). The combined weight of extant evidence for the wellstudied antibody variable (V) gene system only makes sense in terms of an antigen-driven soma-to-germline feedback process involving somatic $\mathrm{V}$ gene retro-transcripts integrating back into homologous $\mathrm{V}$ gene sites in the genomic DNA [25,26]. Apart from endogenous retroviruses [18] what are the likely gene delivery vehicles? Candidates in the male include the incredibly interesting somatic RNA-rich epididymosomes described by the group of Rando $\mathrm{O}$ [27] and the seminal exosome vesicles described by Spadafora $\mathrm{C}$ and coworkers [28].

\section{Ancestral Haplotypes - Adaptation via Shuffling Conserved Pre-Tested Gene Ensembles}

Lamarckian modes of evolution by themselves are manifestly not the sole explanation for the evolutionary phenomenon on Earth. For a more accurate description of metazoan evolution we now need to turn briefly to another important development in evolutionary genomics over the past 35 years - the discovery of ancestral haplotypes (AH), or megabase-long alternative (polymorphic) sequences encoding both information for proteins but also regulation of their expression and thus functional interaction with proteins in other genetic pathways.

$\mathrm{AH}$ sequences encoded at what are known as polymorphic frozen blocks (PFB) were first discovered in the Major Histocompatibility Complex (MHC) by their enigmatic associations with chronic debilitating diseases (autoimmune and metabolic disorders in the main) by Roger Dawkins, Chester Alper, Edmond Yunis and their associates [13,29-32]. One of us (EJS) has recently reviewed the scientific history of these discoveries $[33,34]$.

Polymorphic frozen blocks encoding long ancestral haplotype sequences display features in complete contradiction of the prevailing neo-Darwinian paradigm based on random mutation and selection. Their most striking and controversial property is that each $\mathrm{AH}$ is highly conserved, devoid of both spontaneous mutation and recombination events, and retroviral infections are strongly implicated as a major driver in their genesis $[13,34,35]$. Such PFB are evident in other mammalian species [36] and have been detected at some 108 locations across the human genome [37]. On the latter they are often found in highly duplicated regions encompassing many genes and are peppered with retro-elements and other likely regulatory DNA sequences viz. those potentially encoding transcription factors and their binding domains.

Indeed these features discovered by the Dawkins group in the 1990s led to the Retroviral Induction Model, whereby germline integrated pathogenic retroviruses akin to HIV, would set up many homologous template DNA sequences in the germline, promoting major chromosome rearrangements such as extensive tandem duplications and insertion deletion (indels) events as well as gene copy number (CNV) expansions [13,34,35]. These new gene clusters become focal targets for further retroviral integrations allowing small duplicated clusters to grow over evolutionary time into longer haplotypes containing many imperfectly duplicated segments [35]. In a sense genomic duplications as envisaged by Ohno [38] have been driven by viruses expanding the size of each species' genome.

It appears there has been no real specific gene "selection" for the best part of 50,000-100,000 years of human evolution and expansion except in the context of long haplotypes involving many other genetic regions $[13,35]$. For any given phenotype the ancestral haplotypes located at a "polymorphic frozen block" (PFB) display differences which involve quantitative regulation of the phenotype - where the genes in $\mathrm{AH}$ ensembles mesh like gears in a mechanical transmission system accelerating gene expression up or down [35]. We can now recognize these as quantitative trait loci (QTL) involving immunological, metabolic, cognitive or behavioral features. 
Thus all extant ancestral haplotypes emerging and inherited en bloc from remote ancestors are functionally equivalent in terms of general day-to-day survival; they just differ quantitatively in their outcomes. Adaptations at each generation can be pragmatically achieved by the generational shuffling of $\mathrm{AH}$ at $\mathrm{PFB}$ providing sufficient adaptive polymorphism in the absence of any need for ongoing random mutation and selection. Indeed random mutations and free recombinations are antithetical to the function of ancestral haplotypes which are best viewed as integrated genetic modules conserved (and thus tested) over eons of evolutionary time. However, as discussed, new genetic information can be added to ancestral haplotype backbones provided it is targeted so as not to completely disrupt the stable assemblies [39] - this appears to have happened for the targeted gene conversions at the MHC peptide binding groove sites, and almost certainly occurs in the soma-to-germline transfer of antibody variable gene information into germline V loci [26,34].

The mere existence therefore, of ancestral haplotypes at numerous polymorphic frozen blocks across the human and mammalian genome, poses profound dilemmas for a purely Earth-based Darwinian theory based on random mutation and selection. A new theory of genomic metazoan evolution, one embracing a cosmic context, is clearly required. The processes discussed in this section will certainly not be restricted to species inhabiting the Earth. A connected cosmic biosphere embracing vast cosmological distance scales would be needed to generate an infinite variety of "genes" from which evolving species in terrestrial habitats will be able to select.

\section{Zika Virus - Pragmatic Necessity to Now Accept Panspermian Evolution}

Given this long preamble what then is the conceptual link between the Astronomical Origins of Life, Lamarckian inheritance and the existence of Ancestral Haplotypes? The common feature is they all involve transmissible viruses and their elements, particularly the directly integrating retroviruses - and all are associated with speciation or speciation-like events.

The Earth appears likely to have been regularly infected on cosmic time scales $[7,40]$ with the first appearance of life on the Earth documented to have occurred 3.83-4 billion years ago in the Hadean Epoch when the planet suffered an episode of heavy comet and asteroid impacts [41]. The oceans were formed during this period via the water delivered by comets. Genetic material arriving from space, most likely from comets, would include viruses and cellular genes of bacterial and metazoan origins. In normal evolutionary modes we imagine metazoan lineages of increasing complexity would have emerged and adapted to particular environments, with Lamarckian soma-to-germline mechanisms both direct (RNA- $>$ DNA) and indirect (epigenetic) contributing to these adaptations $[18,24,42]$. In the higher vertebrates, particularly mammals, the localized familial-lineage adaptations would be enhanced by the shuffling of pre-tested and conserved "Ancestral Haplotypes" which we can now view as essentially cosmically generated and then pre-tested polymorphic genetic assembles of virally-related regulatory sequences.

But such processes and local adaptations will not generate new and distinct evolutionary forms required by the fossil record - these processes simply fine-tune existing lineages to their environment and allow adaptations to gradual and non-catastrophic environmental changes (this is the "equilibrium" component of the "punctuated equilibria" pattern recognized in the fossil record by Eldridge and
Gould 40 years ago [4]). New life-forms must be generated by the cosmic viral-induced speciation events strongly implied by all the data assembled by Hoyle, Wickramasinghe and their colleagues these past 40 years [1,2].

\section{So we now return to the Zika virus.}

It is generally agreed that a virus or bacterium that is endogenous to Earth could acquire new characteristics, not only from random mutations, but also by incorporating new genetic sequences from ambient virions. The Zika virus, that is much in the news, appears to have undergone precisely such a change in recent months. Before 2000 the Zika virus was in circulation, but did not cause microencephaly in new born babies - this suggests a major change in the virus. The altered Zika virus, which is now spreading in many countries via a mosquito vector, has been found to affect fetuses in pregnant women, causing babies to be born with reduced brain and skull size. It is also of interest to note that at least one case of transfer of the virus to gametes has been noted in an infected male. The isolation of the virus in semen may be an indication of the soma-to-germline feedback process already occurring in this instance. More are to be expected, much like the peculiar and unexpected seminal transmission mode of HIV when it exploded on the scene unexpectedly in 1981. This epidemic, if it proceeds unchecked, will eventually lead to the emergence of a new human phenotype with reduced brain size and greatly diminished cognitive capacity. It is to be hoped, however, that modern medical science will intervene in time to prevent such a tragic outcome.

We note in this connection that the human brain has seen dramatic changes of volume in the past. Between 2 million and 500,000 years ago, skull volumes in human skeletons appear to have doubled, possibly in several discrete steps [43]. Over the same period it seems likely that our cognitive abilities including the development of speech with the acquisition of the FOXP2 genes had grown $[44,45]$. In all these acquisitions we might assume they have been integrated into preexisting ancestral haplotype assemblies akin to the targeted integration processes already discussed.

Villarreal [44] and Ryan [45] have shown that viral footprints can be identified in human brain tissue to mark important steps that led up to its present condition. The possibility that Zika-virus induced microencephalitis might represent a retrogression of this trend is an alarming prospect that medical science will have to avert before it is too late.

The caveat of course is that objective scientists not only exist but are able to confront the accumulated data and make up their own mind rather than be persuaded by financial necessity, gossip, rumour and fashion. On this important issue, confronting and evaluating the data cannot be avoided. Churchill's famous dictum really does apply in matters like this:

"The truth is incontrovertible. Malice may attack it, ignorance may deride it, but in the end, there it is."

\section{Note}

On February 102016 two separate reports confirmed that the current Zika Virus in circulation in South America is the causative agent of microcephaly and infections in brain and placenta tissues of congenitally infected newborns (Mlakar J, Korva M, Tul N, et al. Zika Virus Associated with Microcephaly New Engl J Med February 10, 2016, at NEJM.org. DOI: 10.1056/NEJMoa1600651; Martines RB, Bhatnagar J, Keating MK, et al. Notes from the Field: Evidence of Zika 
Virus Infection in Brain and Placental Tissues from Two Congenitally Infected Newborns and Two Fetal Losses - Brazil, 2015. MMWR Morb Mortal Wkly Rep 2016;65(Early Release): 1-2. DOI: http:// dx.doi.org/10.15585/mmwr.mm6506eler). With respect to soma-togermline transfer a recent paper was overlooked showing the phenomenon for regulatory double stranded RNA triggering RNA interference (RNAi) in C.elegans (Devanapally S, Ravikumar S, Jose AM. Double-stranded RNA made in C. elegans neurons canenter the germline and cause transgenerational gene silencing Proc. Natl Acad. Sci. USA (2016) 112: 2133-2138 www.pnas.org/cgi/doi/10.1073/pnas. 1423333112).

\section{References}

1. Wickramasinghe NC, Tokoro G, Wainwright M (2015) The Transition from Earth-centred Biology to Cosmic Life. J Astrobiol Outreach 3: 122.

2. Wickramasinghe C (2015) The Search for Our Cosmic Ancestry. World Scientific, Singapore.

3. Joseph R (2009) The evolution of life from other planets. Journal of Cosmology 1: 100-150.

4. Gould SJ, Eldredge N (1977) Punctuated equilibria: the tempo and mode of evolution reconsidered. Paleobiology 3: 115-151.

5. Wickramasinghe NC (2012) DNA sequencing and predictions of the cosmic theory of life. Astrophys Space Sci 343: 1-5.

6. Kopparapu RK (2013) A revised estimate of the occurrence rate of terrestrial planets in rthe habitable zones around kepler m-dwarfs. Astrophys J Lett 767: L8

7. Hoyle F, Wickramasinghe C (1979) Diseases from space. J M Dent \& Sons Ltd., London.

8. Weinstein L (1976) Influenza - 1918, a Revisit? New Engl J Med 294: 1058-1060.

9. Hoyle F, Wickramasinghe C (1977) Does epidemic disease come from space? New Scientist 17 November 1977.

10. Taubenberger JK, Morens DM (2006) 1918 Influenza: The Mother of All Pandemics. Emerging Infectious Diseases $12: 15-22$.

11. Cheung KF, Leung PC (2007) What happened in China during the 1918 influenza pandemic? Int J Infect Dis 11: 360-364.

12. Wickramasinghe C, Wainwright M, Narlikar J (2003) SARS--a clue to its origins? Lancet 361: 1832.

13. Dawkins R, Leelayuwat C, Gaudieri S, Tay G, Jennie Hui, et al. (1999) Genomics of the major histocompatibility complex: haplotypes, duplication, retroviruses and disease. Immunol Rev 167: 275-304.

14. Katzourakis A, Gifford RJ (2010) Endogenous Viral Elements in Animal Genomes. PLoS Genet 6: e1001191.

15. Katzourakis A, Aswad A (2014) The origins of giant viruses, virophages and their relatives in host genomes. BMC Biology 12: 51.

16. Tanne E, Sela I (2005) Occurrence of a DNA sequence of a non-retro RNA virus in a host plant genome and its expression: evidence for recombination between viral and host RNAs. Virology 332: 614-622.

17. Mattick JS (2007) A new paradigm for developmental biology. J Exp Biol 210: 1526-1547.

18. Steele EJ (1979) Somatic Selection and Adaptive Evolution. WilliamsWallace, Toronto.

19. Gorczynski RM, Steele EJ (1980) Inheritance of acquired immunologic tolerance to foreign histocompatibility antigens in mice. Proc Natl Acad Sci USA 77: 2871-2875.

20. Gorczynski RM, Steele EJ (1981) Simultaneous yet independent inheritance of somatically acquired tolerance to two distinct $\mathrm{H}-2$ antigenic haplotype determinants in mice. Nature 289: 678-681.

21. Steele EJ, Pollard JW (1987) Hypothesis : Somatic Hypermutation by gene conversion via the error prone DNA -to- RNA -to- DNA information loop. Molec Immunol 24 : 667-673.
22. Both GW, Taylor L, Pollard JW, Steele EJ (1990) Distribution of mutations around rearranged heavy-chain antibody variable-region genes. Mol Cell Biol 10: 5187-5196.

23. Blanden RV, Rothenfluh HS, Zylstra P, Weiller Gf, Steele EJ (1998 ) The signature of somatic hypermutation appears to be written into the germline IgV segment repertoire. Immunol Rev 162 : 117-132.

24. Steele EJ, Lindley RA, Blanden RV. (1998) Lamarck's Signature: How Retrogenes are Changing Darwin's Natural Selection Paradigm. AllenUnwin, Sydney.

25. Steele EJ (2009) Mechanism of somatic hypermutation: Critical analysis of strand biased mutation signatures at A:T and G:C base pairs. Molec Immunol $46: 305-320$.

26. Steele EJ, Lloyd SS (2015) Soma-to-germline feedback is implied by the extreme polymorphism at IGHV relative to MHC. BioEssays 37 :557-569.

27. Sharma U, Conine CC, Shea JM, Boskovic A, Derr AG, et al. (2015) Biogenesis and function of tRNA Fragments during sperm maturation and fertilization in mammals. Science 351: 391-396.

28. Cossetti C, Lugini L, Astrologo L, Saggio I, Fais S, et al. (2014) Soma-togermline transmission of RNA in mice xenografted with human tumour cells: possible transport by exosomes. PLoS ONE 9: e101629.

29. Dawkins RL, Christiansen FT, Kay PH, Garlepp MJ, McCluskey J, et al. (1983) Disease associations with complotypes, supratypes and haplotypes. Immunol Rev 70:1-22.

30. Awdeh ZL, Raum D, Yunis EJ, Alper CA (1983) Extended HLA/ complement allele haplotypes: evidence for T/t-like complex in man. Proc Natl Acad Sci USA 80: 259-263.

31. Degli-Esposti MA, Leaver AL, Christiansen FT, Witt CS, Abraham LJ, et al. (1992) Ancestral haplotypes: conserved population MHC haplotypes. Hum Immunol 34: 242-252.

32. Alper CA, Larsen CE, Dubey DP, Awdeh ZL, Fici DA, et al 2006 The haplotype structure of the human major histocompatibility complex. Hum Immunol 67: 73-84.

33. Steele EJ (2014) Reflections on Ancestral Haplotypes - Significance for Medical Genomics, Evolution and Human Individuality. Perspectives in Biology and Medicine. 57: 179-197.

34. Steele EJ (2015) Ancestral Haplotypes: Our Genomes Have Been Shaped in the Deep Past. Nearurban Publishing, Dallas, Texas.

35. Dawkins RL (2015) Adapting Genetics: Quantal Evolution After Natural Selection. Nearurban Publishing. Dallas, Texas.

36. Williamson JF, Steele EJ, Lester S, Kalai O, Millman JA, et al. (2011) Genomic evolution in domestic cattle: Ancestral haplotypes and healthy beef. Genomics 97: 304-312.

37. McLure CA, Hinchliffe P, Lester S, Williamson JF, Millman JA, et al. (2013) Genomic evolution and polymorphism: segmental duplications and haplotypes at 108 regions on 21 chromosomes. Genomics 102: 15-26.

38. Ohno S (1970) Evolution by gene duplication. George Allen and Unwin, London.

39. Saunders PT, Ho MW (1976) On the increase in complexity in evolution. J Theor Biol 63: 375-384.

40. Hoyle F and Wickramasinghe C (1981) Evolution from Space. JM Dent, London.

41. Penrose R (2010) Cycles of Time: an extraordinary new view of the universe. Knopf Doubleday Publishing Group, London.

42. Lindley RA (2011) The Soma: How Our Genes Really Work and How That Changes Everything. CYO Foundation, Piara Waters, WA.

43. Hawks J (2013) How has the human brain evolved? Scientific American, 1 July 2013.

44. Villarreal LP (2004) Can Viruses Make Us Human?, Proc. American Phil Soc 148: 296-323.

45. Ryan F (2009) Virolution. Collins. London. 